\title{
Mach-Zehnder Interferometer on the Base of Gradient Index Multimode Interference Structures
}

\author{
M. BŁahut*, D. Kasprzak and M. Sujewicz \\ Department of Optoelectronics, Silesian University of Technology \\ Krzywoustego 2, 44-100 Gliwice, Poland
}

\begin{abstract}
The paper presents the technology and testing measurements of Mach-Zehnder interferometer made by $\mathrm{K}^{+}-\mathrm{Na}^{+}$ion exchange in glass. As a splitter and coupler of measuring and reference arms we used gradient index multimode interference structures in symmetrical configuration. The investigated interferometer, working in asymmetrical configurations, registers the phase shifts resulting from the changes of refractive index of the cover of both interferometer arms.
\end{abstract}

PACS numbers: 42.82.Ds, 42.82.Et, 42.79.Pw

\section{Introduction}

Multimode interference (MMI) structures have been for a few years subject to intensive research studies [1-7]. A large majority of works on MMI concerned interference structures were made on the basis of step-index waveguides. MMI structures can be used to design basic elements of integrated optics having very good optical properties in which the splitting of the input field is effected within a very small area. The properties such as low loss, wavelength insensitivity, small size and uncomplicated fabrication make it possible to find an application of MMI on splitters and couplers, Mach-Zehnder (M-Z) interferometers, phase shifters, modulators, switches and wavelength division multiplexers. Gradient structures made by ion exchange in glass are attractive for MMI technology. Ion exchange technique making use of multi-step diffusion processes, electrodiffusion, heating, diffusive and electrodiffusive burying allows obtaining the modal properties of the obtained waveguides which decide on intermode interference effects to be easily changed. In the papers [2-4] it has been affirmed that self-imaging effects can occur in gradient waveguides produced by $\mathrm{K}^{+}-\mathrm{Na}^{+}$ and $\mathrm{Ag}^{+}-\mathrm{Na}^{+}$ion exchange method, and their applications, among others in the technology of splitters $1 \times 2$ have been presented.

In this paper we present the application of gradient index MMI couplers and splitters in the technology of Mach-Zehnder interferometer in asymmetrical configuration fabricated by $\mathrm{K}^{+}-\mathrm{Na}^{+}$ion exchange in glass.

\section{Operation principles}

The scheme of the examined $\mathrm{M}-\mathrm{Z}$ interferometer is shown in Fig. 1. It consists of the input single-mode

\footnotetext{
* corresponding author; e-mail: marek.blahut@polsl.pl
}

waveguide I, multimode waveguides whereof one works as a $1 \times 2 \mathrm{MMI}$ splitter $\mathrm{S}$ and the other as a coupler $\mathrm{C}$ which are joined together by a curved measuring arm $\mathrm{M}$ and a straight reference arm $\mathrm{R}$, and the single-mode output waveguide $\mathrm{O}$.

The device is designed for $\mathrm{K}^{+}-\mathrm{Na}^{+}$ion-exchange technology. According to [4], the width of the MMI section is $30 \mu \mathrm{m}$ and the length is $1140 \mu \mathrm{m}$. The widths of the single-mode input and output waveguides and the widths of the interferometer arms are equal to $5 \mu \mathrm{m}$.

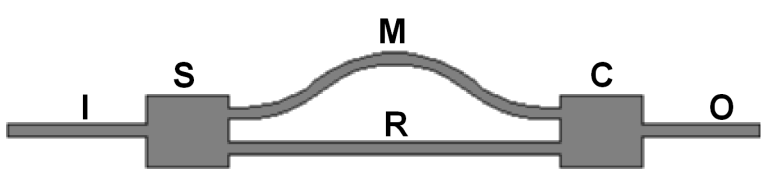

Fig. 1. The scheme of examined $\mathrm{M}-\mathrm{Z}$ interferometer.

The finite difference beam propagation method (FD BPM) simulation of the operation of the examined $\mathrm{M}-\mathrm{Z}$ interferometer is shown in Fig. 2. For each step of the propagation a non-linear diffusion equation [4] through the window described in Fig. 1 was solved, and a two-dimensional gradient profile of the refractive index distribution was determined.

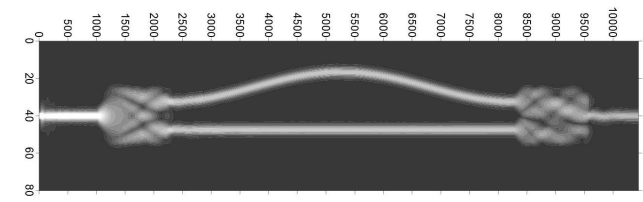

Fig. 2. Numerical simulation by BPM method of the operation of $\mathrm{M}-\mathrm{Z}$ interferometer. The MMI section width is $30 \mu \mathrm{m}$, length $1140 \mu \mathrm{m}$. The width of interferometer arms is $5 \mu \mathrm{m}$.

In the asymmetrical configuration the phase shift $\Delta \phi$ 
depends on the differences of propagation constants and arm lengths of the measuring (M) and of the reference (R) arms

$$
\Delta \phi=\beta_{\mathrm{M}} L_{\mathrm{M}}-\beta_{\mathrm{R}} L_{\mathrm{R}}
$$

The modification of propagation constants can result from waveguide width differences in the interferometer arms. In such a case we have to assume that the signals reaching the MMI coupler have different amplitudes $A_{\mathrm{M}}$ and $A_{\mathrm{R}}$. It can be easily shown that the output power $I_{\text {out }}$ depends on the amplitudes ratio $n$ in the measuring and in the reference arm

$$
I_{\text {out }}=0.25 I_{\text {in }}\left(1+n^{2}+2 n \cos \Delta \phi\right), \quad n=A_{\mathrm{M}} / A_{\mathrm{R}},(2)
$$
where $I_{\text {in }}$ is the power at the input.

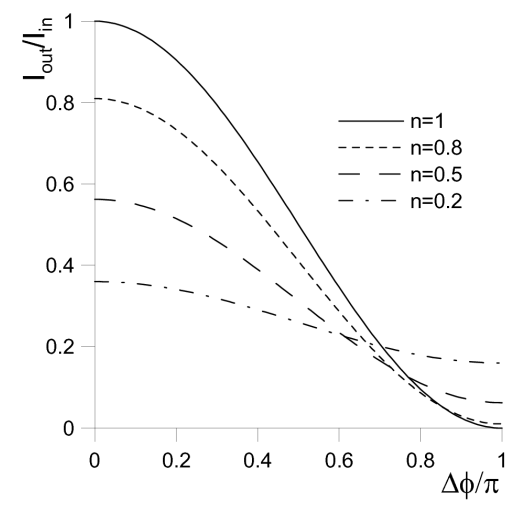

Fig. 3. The dependence of the output power on the phase shift.

In Fig. 3 we show the power at the output as a function of the phase shift $\Delta \phi$ for different amplitude ratios $n$. With the increase of $n$ the depth of signal modulation also increases.

\section{Technology}

The device was fabricated in the diffusion process of $\mathrm{K}^{+}$ions from molten $\mathrm{KNO}_{3}$ as described in [5] over $1 \mathrm{~h}$ and at temperature of $400 \mathrm{~K}$. The curvature of the measuring arm was based on S-bend configuration. To optimize it, we determined numerically by BPM method the dependence of excess losses on the radius of curvature. The characteristic obtained is shown in Fig. 4. Basing on this, we selected the S-bend radius of the waveguide for which the excess losses do not exceed $1 \mathrm{~dB}$.

In the preliminary investigations we used this interferometer to register the phase shifts resulting from the changes of refractive index of the cover of both interferometer arms. In Fig. 5 we can see the dependence of propagation constants of the measuring arms of the widths of $5 \mu \mathrm{m}$ on the refractive index of the cover obtained by the effective refractive index method. Using this characteristic the arms length difference can be calculated, which yields the phase change equal to $\pi$. For the analyzed structure this arm lengths difference

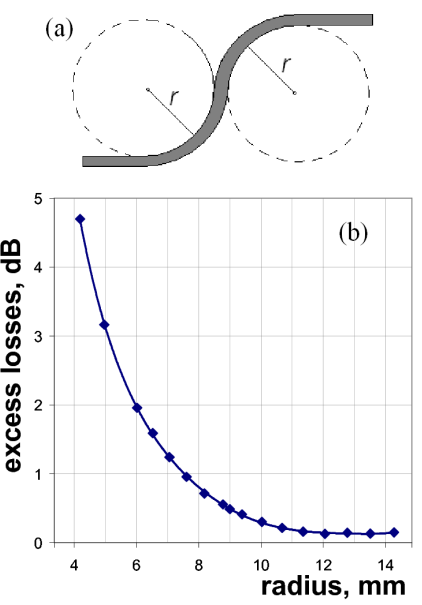

Fig. 4. S-bend geometry: (a) dependence of excess losses on the radius of curvature in curved (S-shape) waveguides, made by $\mathrm{K}^{+}-\mathrm{Na}^{+}$ion exchange (b).

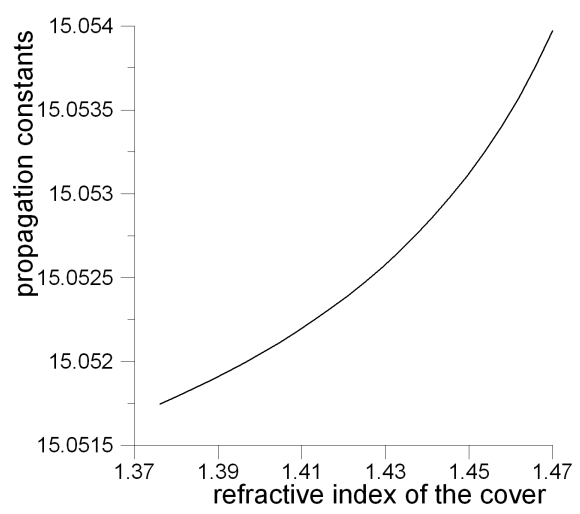

Fig. 5. Dependence of the propagation constant on the refractive index of the cover.

amounts to about $500 \mu \mathrm{m}$. On the basis of it, the interferometer masks were designed. We have fabricated two configurations of $\mathrm{M}-\mathrm{Z}$ interferometers with arm length differences equal to $400 \mu \mathrm{m}$ and $500 \mu \mathrm{m}$. Figure 6 shows the microscopic image of the interferometer masks.

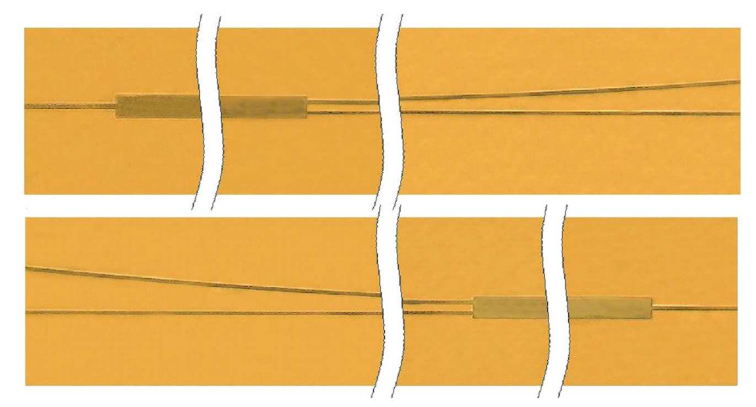

Fig. 6. The microscopic image of masks used. 


\section{Experimental testing results}

The measurements of the characteristics of $\mathrm{M}-\mathrm{Z}$ interferometers - intensity at the output as a function of refractive index of the cover were performed in the experimental stand shown in Fig. 7. M-Z interferometers are excited from a laser by a single-mode fiber. The structures are covered by dielectric substances, water-glycerol solutions, whose refractive index is changed depending on glycerol concentration within the range of 1.37-1.47. The signals at the output are recorded by a CCD camera and registered and analyzed in the computer.

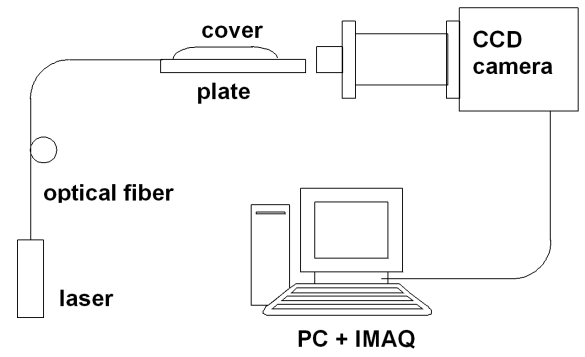

Fig. 7. Scheme of experimental stand.

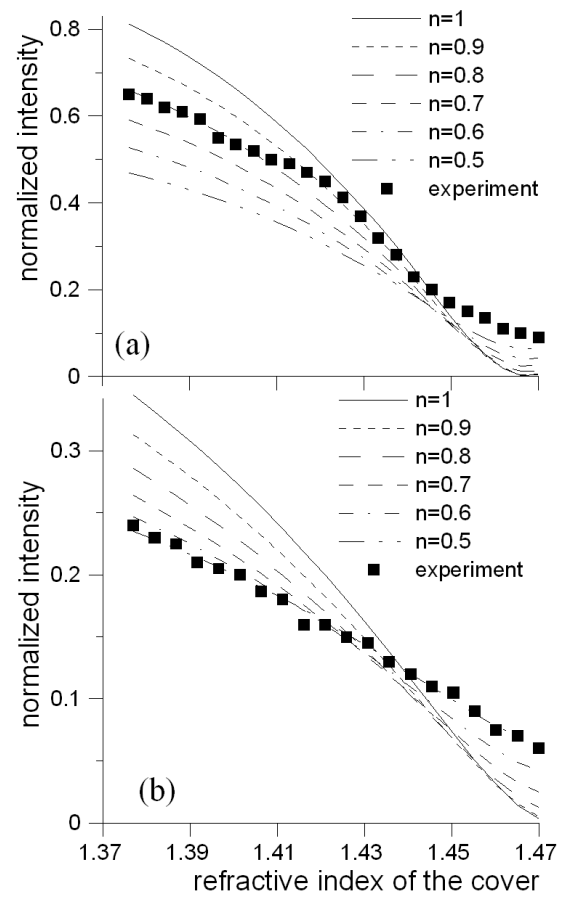

Fig. 8. (a, b) Comparison of experimental and theoretical results for $\mathrm{M}-\mathrm{Z}$ interferometer with arm length difference equal to (a) $500 \mu \mathrm{m}$, (b) $400 \mu \mathrm{m}$.
The preliminary experimental results of the operating characteristics of two interferometer configurations are shown in Fig. 8a,b. The experimental results are compared with the theoretical ones on the basis of Eq. (1) and (2) in which we take into account that one of the signals in the interferometer measuring arms is weaker than the other by $50 \%$ to $90 \%$. As it can be seen, the experimental results are well described by theoretical characteristics for the amplitude ratio $n$ close to 0.7 for Fig. 8a and $n$ equal to 0.5 for Fig. $8 \mathrm{~b}$. As it can be expected, the structure with larger arm length difference is characterized by larger dynamics of its operating characteristic.

\section{Conclusion}

In the paper we have presented theoretical and technological investigations of Mach-Zehnder interferometer made by $\mathrm{K}^{+}-\mathrm{Na}^{+}$ion exchange technology. As a splitter and coupler of the measuring and reference arms we used gradient index multimode interference structures in symmetrical configuration. In the preliminary investigations we used this interferometer to register the phase shifts resulting from the changes of refractive index of the cover of both interferometer arms. Operation characteristics of the device are in a good agreement with the theoretical prediction.

\section{References}

[1] L.B. Soldano, E.C.M. Pennings, J. Lightwave Technol. LT-13, 615 (1995).

[2] M. Błahut, P. Karasiński, D. Kasprzak, R. Rogoziński, Opt. Commun. 214, 47 (2002).

[3] M. Błahut, D. Kasprzak, Opt. Appl. 33, 613 (2003).

[4] M. Błahut, D. Kasprzak, Opt. Appl. 34, 573 (2004).

[5] D. Dorosz, K. Barczak, T. Pustelny, J. Dorosz, Acta Phys. Pol. A 114, A-61 (2008).

[6] T. Pustelny, E. Maciak, Z. Opilski, M. Bednorz, Opt. Appl. 37, 187 (2007).

[7] M. Błahut, A. Opilski, R. Rogoziński, Opt. Appl. 22, 161 (1992). 